POS PROCEEDINGS

\title{
Enlarged Symmetries of Spin Chains and Loop Models
}

\section{Hubert Saleur*}

Service de Physique Théorique CEA Saclay and University of Southern California, Los Angeles

E-mail: hubert.saleurecea.fr; saleurdusc.edu

BETHE ANSATZ: 75 YEARS LATER

October 19-21, 2006

Brussels, Belgium

\footnotetext{
* Speaker.
} 


\section{Introduction}

Despite so many years of work (and so many great papers written) on quantum spin chains, surprises in this area keep enchanting us every now and then. Among those, the most beautiful have to do with symmetry - recent examples include the Yangian symmetry in the Haldane Shastry spin chain [1], or the loop symmetry of the XXZ chain at $q\left(\Delta=\frac{1}{2}\left(q+q^{-1}\right)\right)$ a root of unity [2]. What we shall describe here seems to be yet a new kind of symmetry, whose understanding could well shed new light on logarithmic conformal field theories [3], [4]. Maximum use of this symmetry is most easily mastered with the language of associative algebras. Details appear in [5], while the relation with logarithmic CFTs is discussed in [6]. All the work is being made in collaboration with N. Read.

\section{Oriented loops models and their symmetries}

\subsection{Open case}

To explain the idea and results in the simplest setting, we consider first $\mathrm{SU}(m)$ antiferromagnetic spin chains with open (free) boundary conditions. These chains consist of $2 L$ sites labelled $i=0, \ldots, 2 L-1$, with an $m$-dimensional complex vector space $V_{i} \cong \mathbf{C}^{m}$ at each site $(\mathbf{C}$ is the field of complex numbers). The states can be represented using oscillator operators $b_{i}^{a}, b_{i a}^{\dagger}$ for $i$ even, $\bar{b}_{i a}, \bar{b}_{i}^{a \dagger}$ for $i$ odd, with commutation relations $\left[b_{i}^{a}, b_{j b}^{\dagger}\right]=\delta_{i j} \delta_{b}^{a}(a, b=1, \ldots, m)$, and similarly for $i$ odd. The destruction operators $b_{i}^{a}, \bar{b}_{i a}$ destroy the vacuum state, the daggers indicate the adjoint, and the spaces $V_{i}$ are defined by the constraints

$$
\begin{aligned}
& b_{i a}^{\dagger} b_{i}^{a}=1 \quad(i \text { even }), \\
& \bar{b}_{i}^{a \dagger} \bar{b}_{i a}=1 \quad(i \text { odd })
\end{aligned}
$$

of one boson per site (we use the summation convention for repeated indices of the same type as $a$ ). We define the generators of $\mathrm{U}(m)$ (or in fact of $\mathrm{gl}_{m}$ ) acting in the spaces $V_{i}$ by $J_{i a}^{b}=b_{i a}^{\dagger} b_{i}^{b}$ for $i$ even, $J_{i a}^{b}=-\bar{b}_{i}^{b \dagger} \bar{b}_{i a}$ for $i$ odd, and the commutation relations among the $J_{i}$ s (for each $i$ ) are $i$-independent. Hence the global $\mathrm{gl}_{m}$ algebra, defined by its generators $J_{a}^{b}=\sum_{i} J_{i a}^{b}$, acts in the tensor product $V=\otimes_{i=0}^{2 L-1} V_{i}$ of copies of the fundamental representation of $\mathrm{gl}_{m}$ on even sites, alternating with its dual on odd sites, as desired to construct an antiferromagnetic spin chain. Though the U(1) subalgebra of $\mathrm{gl}_{m}$ generated by $J_{a}^{a}$ acts trivially on the chain (and by a scalar on each site), it is often notationally convenient not to subtract this trace from the generators $J_{a}^{b}$.

The $\mathrm{SU}(m)$-invariant nearest-neighbor coupling in the chain is unique, up to additive and multiplicative constants. It is the usual "Heisenberg coupling" of magnetism, and can be written in terms of operators $e_{i}$, defined explicitly as

$$
e_{i}=\left\{\begin{array}{cl}
\bar{b}_{i+1}^{a \dagger} b_{i a}^{\dagger} b_{i}^{b} \bar{b}_{i+1, b}, & i \text { even }, \\
\bar{b}_{i}^{a \dagger} b_{i+1, a}^{\dagger} b_{i+1}^{b} \bar{b}_{i b}, & i \text { odd. }
\end{array}\right.
$$

The $e_{i}$ 's are Hermitian, $e_{i}^{\dagger}=e_{i}$. Acting in the constrained space $V$, they satisfy $[7,8]$ the relations $[9,10]$

$$
e_{i}^{2}=m e_{i},
$$




$$
\begin{aligned}
e_{i} e_{i \pm 1} e_{i} & =e_{i}, \\
e_{i} e_{j} & =e_{j} e_{i} \quad(j \neq i, i \pm 1) .
\end{aligned}
$$

We write the parameter $m$ as $m=q+q^{-1}$. The abstract associative algebra over the complex numbers $\mathbf{C}$ generated by unity and the $n-1$ generators $e_{0}, \ldots, e_{n-2}$ that satisfy the relations (2.4) (and no other relations algebraically independent of these) with parameter $q \in \mathbf{C}$ will be called the Temperley-Lieb (TL) algebra, $\mathrm{TL}_{n}(q)$ (for $n$ either even or odd). The representation we have constructed in the space $V$ is faithful for $m \geq 2$. All algebras here are over $\mathbf{C}$ and are assumed to include unity.

In the space $V$, a much-studied Hamiltonian for a nearest-neighbor antiferromagnetic spin chain is the $m, \bar{m}$ model with open (free) boundary conditions,

$$
H=-\varepsilon \sum_{i \text { even }} e_{i}-\varepsilon^{-1} \sum_{i \text { odd }} e_{i},
$$

where $\varepsilon>0$ is a parameter; if $\varepsilon \neq 1$, the model is said to have staggered couplings. In the thermodynamic $(L \rightarrow \infty)$ limit a phase transition occurs at $\varepsilon=1$, which is first order for $m>2$, second order for $m=2$. More general Hamiltonians for the spin chain can be constructed, using arbitrary elements of the TL algebra, that is any sum of products of generators, perhaps with random coefficients.

There are also vertex models whose transfer matrices are TL algebra elements. These models may be expanded as configurations of loops that run along (and fill) the edges of the square lattice, with avoided crossings at the vertices. This produces a class of loop model. This uses a well-known graphical representation of the TL algebra that has been described by many authors (see e.g. Ref. [10]). In our models, the loops are viewed as oriented, with the fundamental of $\mathrm{gl}_{m}$ running along in the direction of the arrow. We emphasize again that these orientations are fixed, not summed over (they are not dynamical variables). The two ways of conserving directions of arrows on the loops at a vertex represent the action either of $1 \otimes 1$ or of $e_{i}$ for the two sites $i, i+1$ in question. The symmetry algebra we find here determines the multiplicities in the spectrum of any of these models.

The TL algebra arose in studies of the Potts model $[9,10]$, to which Refs. $[7,8]$ thus found a relation of the $\mathrm{SU}(m)$ antiferromagnetic spin chains. In terms of the TL generators $e_{i}$, the $Q$-state Potts model has the same Hamiltonian, and the $e_{i}$ obey the TL algebra with $Q=m^{2}$, but here for all non-negative integers $Q$ [10]. In the Potts model partition function, the loops are the boundaries of Potts clusters [10]. The possibility of using different representation spaces for a given algebra was a main point of TL [9], and we will have much more to say about this below.

Because of the Hermiticity of the generators $e_{i}$ with respect to the positive-definite inner product on the vector space $V$, it follows that for any element $a$ of the algebra, $a^{\dagger}$ is also an element. If such an algebra is finite dimensional, then it is automatically semisimple, which implies that any of its finite-dimensional modules (representations [11]) is also semisimple, that is fully reducible into a finite direct sum of irreducible representations (simple modules) [12, 13]. Because the representation of the TL algebra in $V$ is faithful for $m \geq 2$, this shows that $\mathrm{TL}_{2 L}(q)$ is semisimple for $m \geq 2$ (in fact, this holds for all $q \geq 1$ ) $[14,15,16]$.

Let us now recall some general algebraic results. In an irreducible representation of dimension $N<\infty$ of an algebra $A, A$ acts as the full matrix algebra $M_{N}(\mathbf{C})$ of all complex $N \times N$ matrices. Any 
semisimple algebra $A$ is isomorphic to a finite direct product of such algebras, one for each distinct irreducible (we refer to two irreducible representations as distinct if they are not isomorphic): $A=$ $\prod_{j} M_{N_{j}}(\mathbf{C})$, where $j$ runs over the set of isomorphism classes of representations, of dimensions $N_{j}$. In matrix language, this means that the algebra $A$ is isomorphic to the algebra of all blockdiagonal complex matrices, where the blocks are $N_{j} \times N_{j}$. It follows that the dimension of $A$ is $\operatorname{dim} A=\sum_{j} N_{j}^{2}$. The commutant $B$ of such an algebra $A$ in a finite-dimensional representation $V^{\prime}$ (the commutant is the algebra of all linear transformations of $V^{\prime}$ that commute with all elements of $A$ ) must also be semisimple, by Schur's lemma. If the $j$ th distinct irreducible representation of $A$ occurs in $V^{\prime}$ with multiplicity $M_{j}$, then the full matrix algebra $M_{M_{j}}(\mathbf{C})$ commutes with $A$ acting in the subspace of $V^{\prime}$ spanned by the copies of the $j$ th irreducible. Assuming that $M_{j}>0$ for all $j$, which means that $A$ is represented faithfully in $V^{\prime}$, then there is a one-one correspondence between the isomorphism classes of irreducible representations of $A$ and $B$, and $B$ is the direct product of algebras $M_{M_{j}}(\mathbf{C})$ (so $\operatorname{dim} B=\sum_{j} M_{j}^{2}$ ). In particular, (i) both algebras have the same number of distinct irreducible representations; (ii) the commutant of $B$ in $V^{\prime}$ is $A$ (that is, the double commutant of $A$, which necessarily contains $A$, is in fact equal to $A$ ); (iii) the space $V^{\prime}$ can be decomposed as

$$
V^{\prime}=\bigoplus_{j} \mathbf{C}^{N_{j}} \otimes \mathbf{C}^{M_{j}}
$$

where $\mathbf{C}^{N_{j}}\left(\mathbf{C}^{M_{j}}\right)$ stands for the irreducible representation of $A$ (resp., $B$ ) of dimension $N_{j}$ (resp., $M_{j}$ ). Hence, $\operatorname{dim} V^{\prime}=\sum_{j} N_{j} M_{j}$. Also, (iv) the center of $A$ (i.e. the subalgebra of elements in $A$ that commute with all elements of $A$ ) is also the center of $B$, and both are isomorphic to $\prod_{j} M_{1}(\mathbf{C})$ (i.e. a direct product of one dimensional algebras, one for each $j$, each isomorphic to the complex numbers). This correspondence between representations of $A$ and $B$ is also a simple form of the more general Morita equivalence of algebras (which applies to algebras that are not necessarily semisimple).

We now apply these general results to $\operatorname{TL}_{n}(q)$ acting in $V$, and construct the commutant algebra explicitly. First, we require information about the representations of the $\mathrm{TL}$ algebra $\mathrm{TL}_{n}(q)$ (in this paragraph, we allow $n$ to be odd or even). The $m=2(q=1)$ case of $V$ is instructive. This is

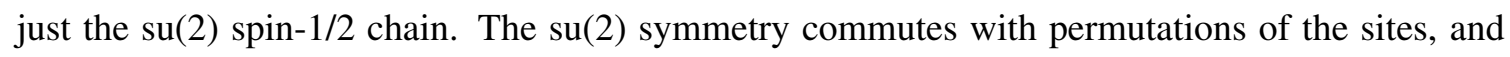
the TL generators are essentially the transpositions of neighbors, which generate the symmetric group $S_{n}$ on $n$ sites. $\mathrm{TL}_{n}(1)$ is isomorphic to the group algebra of $S_{n}$, projected to the space of $S_{n}$ representations that actually occur in $V$. The latter correspond to Ferrers-Young diagrams with $n$ boxes and at most two rows. It follows that the dimension of the $j$ th irreducible representation of $\mathrm{TL}_{n}(1)$, which is the multiplicity of the spin $j$ representation of $\operatorname{su}(2)$ in the chain, is

$$
d_{j}=\left(\begin{array}{c}
n \\
n / 2+j
\end{array}\right)-\left(\begin{array}{c}
n \\
n / 2+j+1
\end{array}\right)
$$

where $n / 2+j$ must be an integer. For $n$ even, $j=0,1, \ldots, n / 2$. The sum of the squares of these dimensions is $\sum_{j} d_{j}^{2}=(n+1)^{-1}\left(\begin{array}{c}2 n \\ n\end{array}\right)$, the dimension of the TL algebra [15]. The same Ferrers-Young diagrams correspond to the representations of su(2) that occur in $V ; 2 j$ equals the difference in the number of boxes in the two rows in the diagram. This well-known decomposition is called [the $\mathrm{su}(2)$ case of] Frobenius-Schur-Weyl duality, and is an example of the correspondence discussed in 
the previous paragraph. For $q>1$, the irreducible representations of $\mathrm{TL}_{n}(q)$ retain the same dimensions, because the algebra varies continuously with $q[14,15,16]$, and hence the TL algebra also retains the same dimension $\operatorname{dim} \operatorname{TL}_{n}(q)=(n+1)^{-1}\left(\begin{array}{c}2 n \\ n\end{array}\right)$. This formula is valid for all $m$, including $m=0$, as can be readily seen from the diagrammatic definition of TL, in which each element of a linear basis corresponds to a diagram $[14,15,16]$.

We now describe the decomposition of our chain $V$ into irreducibles of $\operatorname{TL}_{n}(q)$, for $n=2 L$. We use the following non-orthogonal, but linearly-independent basis states. Each basis state corresponds to a pattern of nested parentheses and dots, such as ()$\bullet(()) \bullet$, with one symbol for each site of the chain ( $2 L=8$ in the example). The parentheses must obey the usual typographical rules for nesting, so that each "(" corresponds to exactly one ")". Also, the dots must not be inside of any parentheses. These rules imply that the () pairs consist of one even and one odd site, and that dots are alternately on even and odd sites, starting with an even site at the left. The states in the chain represented by such a diagram are constructed by contracting the sites that correspond to each () pair into an $\mathrm{SU}(\mathrm{m})$ singlet ("valence bond"). For the dots, the state in the tensor product of spaces $\mathbf{C}^{m}$ (each of which corresponds to a dot) must be chosen so that application of the projection operator to the $\mathrm{SU}(m)$ singlet for any two dots that are adjacent (when parentheses are ignored) annihilates the state. Thus, those sites are "non-contractible".

It is easily seen that the TL algebra applied to these basis states does not mix states with different numbers of non-contractible sites. Application of an $e_{i}$ always produces a valence bond at $i, i+1$, together with a rearrangement of some other contractions for sites that were contracted with $i$ or $i+1$ before (if one of $i, i+1$ was a non-contractible dot, it is moved to another position). Thus, the TL generators $e_{i}$ change a pattern to another valid pattern. However, when $i, i+1$ are both non-contractible, $e_{i}$ annihilates the state. The TL algebra never changes the state on the sequence of dots. The number of valid patterns is independent of $m$, and one can use the $m=2$ case to count them; in this case there is an invertible mapping of the space of states, commuting with the action of $\mathrm{su}(2)$, that maps basis states corresponding to valid patterns with $2 j$ dots to those for "standard" Young tableaus with at most two rows, such that the difference in length of the two rows is $2 j$ (a standard tableau is a Ferrers-Young diagram with one of the numbers $1,2, \ldots, n$ inserted in each box, such that the numbers are increasing both to the right along the rows and down the columns). Hence for each number $2 j=0,2, \ldots, 2 L$ of dots, the number of valid patterns coincides with the dimensions $d_{j}$ of $S_{2 L}$ representations [17]. The basis states are linearly independent and span the $j$ th irreducible representation of $\mathrm{TL}_{2 L}(q)$.

The number of states for each valid pattern with $2 j$ dots determines the dimension $D_{j}$ of the $j$ th representation of the commutant of $\operatorname{TL}_{2 L}(q)$ in $V$. These numbers can be found inductively, by adding another pair of non-contractible dots to the end of a sequence, and are independent of $L$. This leads easily to the recurrence relation [18]

$$
D_{1} D_{j}=D_{j+1}+D_{j}+D_{j-1}
$$

Also, it is clear that $D_{0}=1, D_{1}=m^{2}-1\left[D_{1}\right.$ is the dimension of the adjoint representation of $\mathrm{SU}(m)$ ]. Using $m=q+q^{-1}$, the solution is

$$
D_{j}=[2 j+1]_{q},
$$


where $[n]_{q}=q^{n-1}+q^{n-3}+\ldots+q^{-n+1}=\left(q^{n}-q^{-n}\right) /\left(q-q^{-1}\right)$ is the $q$-deformation of any integer $n$. As a check, the total number of linearly-independent states we constructed is

$$
\sum_{j=0}^{L} D_{j} d_{j}=\left(q+q^{-1}\right)^{2 L}=m^{2 L},
$$

which is exactly $\operatorname{dim} V$. Note that these dimensions are the multiplicities of energy eigenvalues for the generic Hamiltonians in the TL algebra, mentioned earlier. For $m>2$, the dimensions $D_{j}$ asymptotically increase exponentially with $j$. For example, for $m=3$, the first few are 1, 8, 55, 377, $\ldots$, and are the Fibonacci numbers $D_{j}=F_{4 j+2}$. The $m=3$ cases were found for Hamiltonian (2.5) previously [19]. For $j>1$, the decomposition of these multiplets into irreducible representations of $\mathrm{su}(m)$ become increasingly complicated.

\subsection{The explicit commutant}

To construct the commutant algebra explicitly, we introduce the operators (for $k \leq 2 L$ )

$$
\widetilde{J}_{b_{1} b_{2} \ldots b_{k}}^{a_{1} a_{2} \ldots a_{k}}=\sum_{0 \leq i_{1}<i_{2}<\cdots<i_{k} \leq 2 L-1} J_{i_{1} b_{1}}^{a_{1}} J_{i_{2} b_{2}}^{a_{2}} \cdots J_{i_{k} b_{k}}^{a_{k}}
$$

(for $k=0$, we define $\widetilde{J}=1$, and for $k=1, \widetilde{J}_{b}^{a}=J_{b}^{a}$ as defined earlier). For each $k=0,1, \ldots$, these span a space of dimension $m^{2 k}$. In this space of operators we can impose linear conditions, that the contraction of one of the indices $a$ with a neighboring index $b$ [i.e. of $a_{l}$ with $b_{l+1}$ (resp., $b_{l-1}$ ), for $l=1,2, \ldots, k-1$ (resp., $l=2, \ldots, k)]$ is zero. This gives us a basis set $J_{b_{1} \ldots b_{k}}^{a_{1} \ldots a_{k}}$, that are "traceless" in this sense. For example, for $k=2$, we have

$$
J_{b_{1} b_{2}}^{a_{1} a_{2}}=\widetilde{J}_{b_{1} b_{2}}^{a_{1} a_{2}}-\frac{1}{m} \widetilde{J}_{b_{1} a}^{a a_{2}} \delta_{b_{2}}^{a_{1}}-\frac{1}{m} \widetilde{J}_{b b_{2}}^{a_{1} b} \delta_{b_{1}}^{a_{2}}+\frac{1}{m^{2}} \widetilde{J}_{b a}^{a b} \delta_{b_{2}}^{a_{1}} \delta_{b_{1}}^{a_{2}}
$$

and these span a space of dimension $\left(m^{2}-1\right)^{2}$. In general, the dimension is $\left(D_{k / 2}\right)^{2}$. The exact forms are

$$
J_{b_{1} b_{2} \ldots b_{k}}^{a_{1} a_{2} \ldots a_{k}}=\left(P^{\bullet} P_{\bullet} \widetilde{J}\right)_{b_{1} b_{2} \ldots b_{k}}^{a_{1} a_{2} \ldots a_{k}},
$$

where $P^{\bullet}\left(P_{\bullet}\right)$ is the (Jones-Wenzl) projection operator to the "traceless" sector on the vector space indexed by $\left(a_{1}, b_{2}, \ldots\right)$ [resp., $\left.\left(b_{1}, a_{2}, \ldots,\right)\right]$, which can be constructed recursively using the $\operatorname{TL}_{k}(q)$ algebra in these spaces [20].

One can readily show that: (i) all $J_{b_{1} b_{2} \ldots b_{k}}^{a_{1} a_{2} \ldots a_{k}}$ commute with all the $e_{i}$, hence with all of $\operatorname{TL}_{2 L}(q)$ (they leave the patterns unchanged); (ii) all $J_{b_{1} b_{2} \ldots b_{k}}^{a_{1} a_{2} \ldots a_{k}}$ with $k>2 j$ annihilate the $j$ th irreducible representation of the commutant algebra; (iii) the space of $J_{b_{1} b_{2} \ldots b_{k}}^{a_{1} a_{2} \ldots a_{k}}$ s with $k=2 j$ acts as the matrix algebra $M_{D_{j}}(\mathbf{C})$ on the $j$ th irreducible representation; (iv) $J_{b_{1} b_{2} \ldots b_{k}}^{a_{2} \ldots b_{k}}$ with $k<2 j$ map the $j$ th irreducible representation into itself, and hence in that subspace can be written as linear combinations of those with $k=2 j$. In particular, in our chain of $2 L$ sites, the operators with $k$ odd are linear combinations of those with $k$ even. Hence only even $k$ are needed. These results show that the algebra spanned by $J_{b_{1} b_{2} \ldots b_{k}}^{a_{1} a_{2} \ldots a_{k}}(k=0,2, \ldots)$ is the commutant algebra $\mathscr{A}_{m}(2 L)$ of $T L_{2 L}(q)$ in $V$, with dimension $\operatorname{dim} \mathscr{A}_{m}(2 L)=\sum_{j}\left(D_{j}\right)^{2}$. Because the dimensions $D_{j}$ are independent of $L$, the limit $L \rightarrow \infty$ exists, and we write $\mathscr{A}_{m}=\lim _{L \rightarrow \infty} \mathscr{A}_{m}(2 L)$.

The "obvious" global symmetry algebra is $\mathrm{gl}_{m}$, or more accurately the universal enveloping algebra (UEA) $U\left(\mathrm{gl}_{m}\right)$ of $\mathrm{gl}_{m}$, which is the associative algebra generated by the generators $J_{a}^{b}$ of 
$\mathrm{gl}_{m}$, subject to the commutation relations of $\mathrm{gl}_{m}$ [or similarly for $\left.U\left(\mathrm{sl}_{m}\right)\right]$ [21, 22]. For $m>2$, our algebra $\mathscr{A}_{m}$ is strictly larger than $U\left(\mathrm{sl}_{m}\right) ; U\left(\mathrm{sl}_{m}\right)$ is a proper subalgebra of $\mathscr{A}_{m}$ [23], and hence the representations of $\mathscr{A}$ can be decomposed into representations of $\mathrm{sl}_{m}$. The dimension of $\mathscr{A}_{m}(2 L)$ can be found in closed form, and grows exponentially with $L$ :

$$
\begin{aligned}
\operatorname{dim} \mathscr{A}_{m}(2 L) & =\frac{q^{4 L+4}-q^{-4 L-4}}{\left(q-q^{-1}\right)^{2}\left(q^{2}-q^{-2}\right)}-\frac{2(L+1)}{\left(q-q^{-1}\right)^{2}} \\
& =\frac{[2 L+2]_{q^{2}}-(2 L+2)}{\left(q-q^{-1}\right)^{2}} \\
& \sim \frac{q^{4 L}}{\left(1-q^{-2}\right)\left(1-q^{-4}\right)}
\end{aligned}
$$

as $L \rightarrow \infty$; here we used $q>1$. By contrast, the dimension of the quotient of $\mathrm{sl}_{m}$ that acts faithfully in the chain is the sum of squares of the dimensions of irreducibles that occur, and the latter dimensions are known polynomials in the highest weight of the representation, of degree at most $m(m-1) / 2$ (the dimensions of irreducibles of $\mathrm{sl}_{m}$ are found by Frobenius-Schur-Weyl duality, and given by the Weyl dimension formula). The highest weights that occur are bounded by something of order the length $L$ of the chain. We have not made a precise estimate of the dimension of the resulting associative algebra, but it is clear that it is bounded by a polynomial in $L$, and thus much smaller than $\mathscr{A}_{m}(2 L)$ for large $L$.

We do not know of a "small" or "simple" set of generators for $\mathscr{A}_{m}$ (that would be analogous to the set of $J_{b}^{a}$ for $\left.U\left(\mathrm{gl}_{m}\right)\right) . \mathscr{A}_{m}$ is not the Yangian of $\mathrm{sl}_{m}$. However, the properties above imply that $\mathscr{A}_{m}(2 L)$ is a cellular algebra for all $L$, for which we have given a cellular basis $J_{b_{1} b_{2} \ldots b_{k}}^{a_{1} a_{2} \ldots a_{k}}(k=0$, $2, \ldots$ ), in the sense defined in Ref. [24] (for an exposition, see e.g. Ref. [25]). This fact also generalizes to supersymmetric versions we will introduce later.

\subsection{Periodic case}

We generalize the results to the closed (periodic) version of the $\mathrm{SU}(m)$ spin chain models. The space of states is the same (with an even number $2 L$ of sites), and the TL generators are defined as there, but now there are $2 L$ generators $e_{i}$, which obey the relations (2.4) with $i \pm 1$ interpreted cyclically, with $i=2 L \equiv 0(\bmod 2 L)$. In addition, there is now an obvious cyclic symmetry of the system. We can introduce an operator $u^{2}$ (with inverse $u^{-2}$ ) which translates any state to the right by 2 sites (so as to be consistent with the distinction of two types of sites carrying dual representations), so $u^{2 L}=1$ (there are no odd powers of $u$, though there are in the unoriented or $\mathrm{O}(m)$ cases). We have $u^{2} e_{i} u^{-2}=e_{i+2}$. These operators generate an algebra.

The precise algebra can be defined abstractly as an algebra of diagrams as for TL, but this time on an annulus (or finite cylinder), in which a general basis element corresponds to a diagram of $2 L$ sites on the inner, and $2 L$ on the outer boundary; the sites are connected in pairs, but only configurations that can be represented using lines inside the annulus that do not cross are allowed [26]. Further, for the oriented loops models, the lines must be orientable, such that the arrows emanate from the even sites and enter the odd sites on the inner boundary, and the reverse for the outer boundary. Multiplication is defined in a natural way on these diagrams, by joining an inner to an outer annulus, and removing the interior sites [26]. We emphasize that whenever a closed loop is produced when diagrams are multiplied together, this loop must be replaced by a numerical factor 
$m$ (as for the TL algebra), even for loops that wind around the annulus, as well as for those that are homotopic to a point. The algebra is generated by the elements $e_{i}$ and $u^{2}$, and they obey the above relations, which however are not a complete set. (The numerical factor $m$ for winding loops is not a consequence of the stated relations, but a separate assumption.) We call this finite-dimensional "annular" algebra [26] the Jones-TL, or JTL algebra, $\operatorname{JTL}_{2 L}(q)$ [26, 27] (the latter terminology is not standard). It is easily seen that our definitions produce a representation of $\operatorname{JTL}_{2 L}(q)$ in $V$, however it turns out that it is faithful only when $m>2$. For $m=2$, the TL algebra already contains all permutations of the sites, and the extra generators $e_{-1}$ and $u^{ \pm 2}$ acting in $V$ can be expressed in terms of the others. Also, for real $q>0$, the JTL algebra is semisimple only for $q \neq 1$ [26], unlike the TL algebra. We will see that the JTL algebra is much richer than the TL algebra. For other periodic generalizations of the TL algebra, which are infinite dimensional, see e.g. Refs. [28, 29].

On passing from $\operatorname{TL}_{2 L}(q)$ to $\operatorname{JTL}_{2 L}(q)$, some irreducible representations of $\operatorname{TL}_{2 L}(q)$ will combine to form irreducibles of $\mathrm{JTL}_{2 L}(q)$. On the other hand, since we work in the same space $V$, when the algebra becomes larger, its commutant must become smaller, and some irreducible representations of the commutant will break into irreducibles of the commutant of $\operatorname{JTL}_{2 L}(q)$. (These remarks assume the algebras involved are semisimple.)

The dimensions of the irreducible representations of $\operatorname{JTL}_{2 L}(q)$ for $q>1$ are known [26]. We construct representations of the JTL algebra using parentheses and dots again, but now parentheses can be paired cyclically, so $) \bullet(()) \bullet$ ( is a valid pattern (valid patterns may also be defined by drawing them on a disk with the sites on the boundary, and lines within the disk connect contracted sites without crossing, while noncontractible sites can be reached within the disk from one another without crossing a contraction line). Contractions that cross the end of the chain, like one in the preceding example, become pairs of dots if one reverts to the open TL point of view, and so one finds for the number of valid patterns with $2 j$ dots

$$
\widehat{d_{j}}=\sum_{j^{\prime}=j, j+1, \ldots} d_{j^{\prime}}=\left(\begin{array}{c}
2 L \\
L+j
\end{array}\right) .
$$

This is valid for $j>0$. For the $j=0$ case, all contraction lines can be drawn without crossing the $0,2 L-1$ link, so $\widehat{d_{0}}=d_{0}$. [These formulas, which as we will see give the dimensions $\widehat{d_{j}}$ of the irreducible representations of $\operatorname{JTL}_{2 L}(q)$, also show how the representations decompose when considered as representations of the subalgebra $\operatorname{TL}_{2 L}(q)$.]

For the set of valid patterns for each value $j=0,1, \ldots, L$, one has a set of non-orthogonal but linearly independent basis states, by again associating a singlet valence bond to each pair () of corresponding parentheses, and for the non-contractible sites (now defined cyclically), states that vanish if one such site is contracted with its neighbor on either side (cyclically). The subspace spanned by these elements is a representation of $\operatorname{JTL}_{2 L}(q)$ and of its commutant $\widehat{\mathscr{A}_{m}}(2 L)$, and its dimension is $\widehat{d}_{j} \widehat{D}_{j}$, where the dimensions $\widehat{D}_{j}$ for each pattern will now be found. By comparing with the definitions for the open case, we see that the dimensions $\widehat{D}_{j}$ obey $D_{j}=\widehat{D}_{j}+D_{j-1}(j \geq 2)$, $D_{1}=\widehat{D}_{1}, D_{0}=\widehat{D}_{0}$. That is,

$$
\widehat{D}_{j}=\left\{\begin{array}{l}
q^{2 j}+q^{-2 j} \quad(j>1), \\
q^{2}+1+q^{-2} \quad(j=1), \\
1 \quad(j=0)
\end{array}\right.
$$


Note that again $\sum_{j} \widehat{d}_{j} \widehat{D}_{j}=m^{2 L}=\operatorname{dim} V$.

Unlike the open chains, for the closed chains the representations of $\operatorname{JTL}_{2 L}(q) \otimes \widehat{\mathscr{A}_{m}}(2 L)$ of dimension $\widehat{d}_{j} \widehat{D}_{j}$ that we have now constructed for each $j$ are not irreducible when $j \geq 2$. There is a non-trivial center of the restriction of $\operatorname{JTL}_{2 L}(q)$ and of $\widehat{\mathscr{A}_{m}}(2 L)$ to the $j$ th subspace. This may be seen most easily in terms of the commutant $\widehat{\mathscr{A}_{m}}(2 L)$. For any basis state in the $j$ th subspace, the states on the non-contractible sites can be cyclically permuted by moving them two steps to the right, without affecting the pattern. This operation clearly commutes with the JTL algebra, so when viewed as acting on all the basis states simultaneously it gives an operator which lies in the commutant. Further, it commutes with all elements of the commutant (restricted to this subspace), because as we will see in the explicit expressions below, these elements involve sums over position which ensure that they are invariant under these operations on the basis states (ultimately this is because of the isomorphism of the JTL algebra $e_{i} \rightarrow e_{i+1}$ for all $i$ ). Hence this operator lies in the center of the commutant (acting in the $j$ th subspace), and so must also lie in the center of the JTL algebra. By Schur's lemma, it acts as a root of unity $e^{2 i K}$ in any irreducible representation of either algebra. We call $K$ (defined modulo $\pi$ ) the pseudomomentum. As translation of the noncontractible sites by $2 j$ steps brings the state back to itself, we have $j K \equiv 0(\bmod \pi)$. We may conclude that, for each $j \geq 2$, though all irreducible representations of $\operatorname{JTL}_{2 L}(q)$ have the same dimension $\widehat{d}_{j}$, they are not all isomorphic, and there is a distinct irreducible representation for each distinct allowed $K$, and thus $j$ distinct isomorphism classes of irreducibles in all [26].

The representations of the commutant of dimension $\widehat{D}_{j}$ can be decomposed into eigenspaces of $K$, with $K=\pi P / N$ where $P \geq 0$ and $N$ are coprime ( $N$ is a divisor of $j$, written $N \mid j$ ). We will denote the dimensions of these subspaces by $\widehat{D}_{j K}$, with $\sum_{K} \widehat{D}_{j K}=\widehat{D}_{j}$, from which again, $\sum_{j, K} \widehat{D}_{j K} \widehat{d}_{j}=m^{2 L}$. For $j=0,1, K \equiv 0$ and $\widehat{D}_{j K}=\widehat{D}_{j}$. When the state on the sequence of $2 j$ non-contractible sites is periodic with period $d, 1 \leq d<j$ (with $d \mid j$ ), it contributes only to pseudomomenta such that $N \mid d$. Using Möbius inversion [30] (similarly to Appendix A of Ref. [18]), we obtain the dimensions $\widehat{D}_{j K}$ of the representations with $j \geq 2$ and given $K$ of the commutant $\widehat{\mathscr{A}}_{m}(2 L)$ of $\operatorname{JTL}_{2 L}(q)$ for $m>2$,

$$
\widehat{D}_{j K}=\sum_{d, d^{\prime}: N \mid d} \frac{\mu\left(d / d^{\prime}\right)}{d}\left(q^{2 d^{\prime}}+q^{-2 d^{\prime}}\right),
$$

where the sum is over all positive divisors $d, d^{\prime}$ of $j$, and $\mu(x)$ is the Möbius function [30]. Alternatively, by calculating the trace of the projection operator onto pseudomomentum $K$ for a fixed pattern, we obtain

$$
\widehat{D}_{j K}=\frac{1}{j} \sum_{r=0}^{j-1} e^{2 i K r}\left[q^{2(j \wedge r)}+q^{-2(j \wedge r)}\right]
$$

where $j \wedge r$ denotes the highest common divisor of $j$ and $r(j \wedge 0=j$ for all integers $j \geq 0)$. These two expressions are equal, again by using Ref. [30]. These multiplicities were given in the second form by Jones [26] (for these oriented cases, we have corrected a small error at the end of Ref. [26]). These representations of $\widehat{\mathscr{A}_{m}}(2 L)$ are irreducible, and the dimension of the algebra is $\operatorname{dim} \widehat{\mathscr{A}_{m}}(2 L)=\sum_{j, K}\left(\widehat{D}_{j K}\right)^{2}$. In the $L \rightarrow \infty$ limit, we obtain an algebra $\widehat{\mathscr{A}_{m}}=\lim _{L \rightarrow \infty} \widehat{\mathscr{A}_{m}}(2 L)$.

If we put $m=2$ (even though this is a case in which the JTL algebra does not act faithfully in $V)$, the multiplicities correctly vanish whenever $K \not \equiv 0(\bmod \pi)$, but the formula for $\widehat{D}_{j 0}$ for $j>1$ is not correct for this case. Here, because the image of the JTL algebra that acts faithfully in $V$ is the same as TL, its commutant is a quotient of $U\left(\mathrm{sl}_{2}\right)$, with irreducible dimensions $D_{j}=2 j+1$. 
Some elements of $\widehat{\mathscr{A}_{m}}(2 L)$ can be constructed as in the open case. We use, for $k \geq 1$,

$$
\widetilde{J}_{b_{1} b_{2} \ldots b_{k}}^{a_{1} a_{2} \ldots a_{k}}=\sum_{i_{1}<i_{2}<\cdots<i_{k}<i_{1}} J_{i_{1} b_{1}}^{a_{1}} J_{i_{2} b_{2}}^{a_{2}} \cdots J_{i_{k} b_{k}}^{a_{k}}
$$

where the summations extend periodically on the chain; these commute with $u^{2}$. A set of elements of the commutant $\widehat{\mathscr{A}_{m}}(2 L)$ can now be written, for $k$ even, as

$$
\widehat{J}_{b_{1} b_{2} \ldots b_{k}}^{u_{1} a_{2} \ldots a_{k}}=\left(P^{\bullet} P \bullet \widetilde{J}\right)_{b_{1} b_{2} \ldots b_{k}}^{a_{1} a_{2} \ldots a_{k}},
$$

where, similarly to the open case, the projector $P^{\bullet}\left(P_{\bullet}\right)$ is the projection operator to the "traceless" sector (annihilated by all $e_{i}$ ) on the vector space indexed by $\left(a_{1}, b_{2}, \ldots\right)$ [resp., $\left.\left(b_{1}, a_{2}, \ldots,\right)\right]$. The projectors certainly exist, as they project onto (non-irreducible for $k>2$ ) representations of the semisimple algebra $\operatorname{JTL}_{k}(q)$. For $k$ odd, there is another set,

$$
\widehat{J}_{b_{1} b_{2} \ldots b_{k}}^{a_{1} a_{2} \ldots a_{k}}=(P \bullet \widetilde{J})_{b_{1} b_{2} \ldots b_{k}}^{a_{1} a_{2} \ldots a_{k}}
$$

in which $P_{\bullet}^{\bullet}$ is the projection operator to the traceless sector (annihilated by $e_{i}$ ) on the single vector space indexed by $\left(a_{1}, b_{2}, a_{3}, \ldots, a_{k}, b_{1}, a_{2}, \ldots, b_{k}\right)$. These projectors exist in the algebra $\operatorname{JTL}_{2 k}(q)$. (For $k=1, \widehat{J_{b}^{a}}$ is the traceless generator of $\mathrm{sl}_{m}$.) The $\widehat{J_{\mathrm{s}}}$ have the cyclic invariance property,

$$
\widehat{J}_{b_{1} b_{2} \ldots b_{k}}^{a_{1} a_{2} \ldots a_{k}}=\widehat{J}_{b_{k} b_{1} \ldots b_{k-1}}^{a_{k} a_{1} \ldots a_{k-1}}
$$

(the cyclic property is clear for the $\widetilde{J_{\mathrm{s}}}$, and for the $\widehat{J_{\mathrm{S}}}$ with $k$ even uses the fact that the matrix elements of the projectors $P_{\bullet}, P^{\bullet}$ are the same real numbers when written out in the respective bases).

Unlike the open case, for the closed case the $\widehat{J}$ operators with $k$ even do not form a linear basis for $\widehat{\mathscr{A}_{m}}(2 L)$; however, they do generate it. Let us study how they act on the $j, K$ irreducible representations for $k=2 j$. A natural decomposition of this space of $\widehat{J_{\mathrm{S}}}$ is obtained by using the projector $P_{(K)}^{\bullet}\left[\right.$ and $\left.P_{\bullet}^{(-K)}\right]$ onto the subspace of the space indexed by $\left(a_{1}, b_{2}, \ldots\right)\left[\right.$ resp., $\left.\left(b_{1}, a_{2}, \ldots,\right)\right]$ that has pseudomomentum $K$ (resp., $-K$ ) as well as being annihilated by all $e_{i}$. This is possible because the $\widehat{J_{\mathrm{S}}}$ do preserve pseudomomentum. We note that the cyclic invariance property implies that these operators for $K$ and $-K$ are the same (up to some relabelling). We choose an orthonormal basis for this subspace, indexed by $\alpha, \beta, \gamma, \ldots$ (resp., $\alpha^{*}, \ldots$; there is a correspondence between these bases as indicated by the notation), and write these projected $\widehat{J_{\mathrm{S}}}$ as $\widehat{J}_{\alpha \beta}$. Among the irreducibles with $j=k / 2$, these operators annihilate those with pseudomomentum $\not \equiv K$ or $-K(\bmod \pi)$. Strictly speaking, the cases in which $K \equiv-K(\bmod \pi)$ should be distinguished from the more general cases $K \not \equiv-K(\bmod \pi)$; we will return to this after dealing with the generic case. Then in general one finds that $\widehat{J_{\alpha \beta}}$ maps $\beta$ (with pseudomomentum $K$ ) onto $\alpha$, but also $\alpha^{*}$ (with pseudomomentum $-K$ ) to $\beta^{*}$. We may re-normalize such that the first of these non-zero matrix elements is equal to one. Now define operators $\mathscr{J}_{\alpha \beta}=\widehat{J}_{\alpha \gamma} \widehat{J}_{\gamma \beta}$ where $\alpha \neq \beta$, and hence also $\alpha^{*} \neq \beta^{*}$ (the summation convention is not in force for the Greek indices). This is possible because all the spaces for $j>0$ have dimension $>1$. Then we see that $\mathscr{J}_{\alpha \beta}$ acts as the elementary matrix $E_{\alpha \beta}$ (whose only non-zero entry is 1 in position $\alpha, \beta$ ) in the pseudomomentum $K$ representation, and annihilates all others with $j=k / 2$, including that for $-K$. Finally the diagonal entries are defined as $\mathscr{J}_{\alpha \alpha}=\mathscr{J}_{\alpha \beta} \mathscr{J}_{\beta \alpha}$, (for any $\beta \neq \alpha$ ) which acts as $E_{\alpha \alpha}$ in the $j, K$ irreducible. For the cases in 
which $K \equiv-K(\bmod \pi)$, one should note that the basis states $\alpha^{*}$ are in the same space, and are essentially a permutation of the basis labelled $\alpha . \mathscr{J}_{\alpha \beta}=\widehat{J}_{\alpha \gamma} \widehat{\widehat{J}}_{\gamma \beta}$ acts as $E_{\alpha \beta}$ only if $\alpha^{*}, \beta^{*}$ and $\gamma$ are all distinct. This works provided the space has dimension $>2$, which they all do (for $j>0$ ). As the elementary matrices form a linear basis for the full matrix algebra $M_{\widehat{D}_{j K}}(\mathbf{C})$, we have shown that the operators $\widehat{J}$ do generate the algebra $\widehat{\mathscr{A}_{m}}(2 L)$.

Thus finally, we have defined a set of operators $\mathscr{J}_{\alpha \beta}$ for all even $k$ and for all allowed $K$ (mod $\pi$ ), which are a linear basis for $\widehat{\mathscr{A}_{m}}(2 L)$, and which act on the corresponding $(j=k / 2)$ irreducible as elementary symmetric matrices, while annihilating those with the same $j$ but different $K$, as well as those with $j<k / 2$. Once again, these properties imply that this basis is cellular [24, 25].

\section{Supersymmetric spin chains}

We now briefly describe the results for the supersymmetric generalizations of the spin chains (such as those that occur in the spin quantum Hall effect [31]). The spin chains for the oriented loops models can be generalized so that each site carries a $\mathbf{Z}_{2}$-graded vector space of dimensions $m+n$ for the even (bosonic), $n$ for the odd (fermionic), subspace ( $n \geq 0$ is an integer). This space is the fundamental of the Lie superalgebra $\operatorname{gl}(m+n \mid n)$ for $i$ even, and its dual for $i$ odd. The chain is the graded tensor product of these $V_{i}$ (it may be constructed [18] using fermion operators $f_{i a}^{\dagger}, \bar{f}_{i}^{a \dagger}$ for $a=m+n+1, \ldots, m+2 n$, while $a=1, \ldots, m+n$ corresponds to boson operators as in the $n=0$ special case. The (J)TL algebra is again generated by operators $e_{i}$ (and $u^{2}$ ). These models exist for all integer $m$, provided $m+n, n \geq 0$ [32], and are non-trivial when $m+2 n>1$. The phase transition properties, including scaling dimensions, are the same independent of $n$, though some multiplicities may vanish for small $n$. Even though the finite-dimensional representations of $\operatorname{gl}(m+n \mid n)$ are not always semisimple, the representations of $\mathrm{TL}_{2 L}(q)$ and its commutant $\mathscr{A}_{m+n \mid n}(2 L)$ are still semisimple for $|m| \geq 2$, and similarly for $\operatorname{JTL}_{2 L}(q)$ and its commutant $\widehat{\mathscr{A}_{m}+n \mid n}(2 L)$ for $|m|>2$. (The commutant algebras here are actually superalgebras when $n>0$; details about graded tensor products and superalgebras can be found in the [5].) The notation involving $m, n$ for these chains will be used consistently from here on.

For the semisimple cases, the preceding constructions can be carried through for all $n \geq 0$, with only minor variations. The dimensions of the irreducible representations of the commutants can be generalized to the total (usual) dimension, and the superdimension (sdim) which is the dimension of the even (bosonic) subspace minus the dimension of the odd (fermionic) subspace. The super-dimensions will now be denoted $D_{j}$ or $\widehat{D}_{j K}$, as they are determined by $m$ alone (in fact, by $m^{2}$ ), independent of $n$, and are given by the same formulas as above, which were the $n=0$ special cases. The total dimensions will be denoted $D_{j}^{\prime}$ or $\widehat{D}_{j K}^{\prime}$, and involve also $q^{\prime}$ determined by $m+2 n=q^{\prime}+q^{\prime-1}$. For the open case, the total dimensions are given by the same form $D_{j}^{\prime}=$ $[2 j+1]_{q^{\prime}}[18]$. For the closed case, total dimensions can be obtained by calculating the trace of the projection operator onto pseudomomentum $K$ for a fixed pattern. One must be careful of minus signs that arise when an odd state of a segment of the non-contractible sites is translated around the system. For $j>1$, the total dimensions for $|m|>2$ are

$$
\widehat{D}_{j K}^{\prime}=\frac{1}{j} \sum_{r=0}^{j-1} e^{2 i K r} w(j, j \wedge r),
$$


where $j \wedge r$ denotes the highest common divisor of $j$ and $r(j \wedge 0=j$ for all integers $j \geq 0)$, and

$$
w(j, d)=\left(q^{2 d}+q^{-2 d}\right) \delta_{j / d \equiv 0}+\left(q^{2 d}+q^{\prime-2 d}\right) \delta_{j / d \equiv 1},
$$

where again $d \mid j$, and the congruences are modulo 2. For $n=0$ this clearly reduces to eq. (2.20) [26]. The general case can be simplified and (using formulas from Ref. [30]) shown to be equal to the numbers $\Lambda_{\bmod }(M=j, N)$ for these models obtained by a different method in Ref. [18] (up to a continuation to the different range $|m| \leq 2$ studied there). For $j=0,1$, we have $K \equiv 0$ only, and $\widehat{D}_{j 0}^{\prime}=1, q^{\prime 2}+1+q^{\prime-2}$, respectively [the dimensions of the singlet and the adjoint of $\left.\operatorname{sl}(m+n \mid n)\right]$.

Finally, we give details of our construction for the open $\operatorname{gl}(1 \mid 1)$ spin chain, which is a free fermion system [18]. The free fermion form of the model is defined using fermion operators $f_{i}$ and their adjoints $f_{i}^{\dagger}, i=0,1, \ldots, 2 L-1$, which obey $\left\{f_{i}, f_{i^{\prime}}\right\}=0,\left\{f_{i}, f_{i^{\prime}}^{\dagger}\right\}=(-1)^{i} \delta_{i i^{\prime}}$. The TL generators can be written as

$$
e_{i}=\left(f_{i}^{\dagger}+f_{i+1}^{\dagger}\right)\left(f_{i}+f_{i+1}\right)
$$

for $i=0, \ldots, 2 L-2$. Some symmetry operators in $J^{(k)}$, for $k=1,2$, which commute with all the $e_{i} \mathrm{~s}$, are

$$
\begin{aligned}
F & =\sum_{i} f_{i}, \\
F^{\dagger} & =\sum_{i} f_{i}^{\dagger}, \\
F_{(2)} & =\sum_{i<i^{\prime}} f_{i} f_{i^{\prime}}, \\
F_{(2)}^{\dagger} & =\sum_{i<i^{\prime}} f_{i^{\prime}}^{\dagger} f_{i}^{\dagger}, \\
N & =\sum_{i}(-1)^{i} f_{i}^{\dagger} f_{i}-L .
\end{aligned}
$$

The remaining symmetry operators turn out to be sums of products of these, so this set of five operators (together with 1 ) is a set of generators of the full algebra $\mathscr{A}_{1 \mid 1}$. The graded commutators of these five close on themselves, so they form a Lie superalgebra. $F, F^{\dagger}$ generate a Lie subsuperalgebra isomorphic to $\operatorname{psl}(1 \mid 1) . F_{(2)}, F_{(2)}^{\dagger}$, and $N$ generate an $\mathrm{sl}_{2}$ Lie subalgebra, with $N$ as $2 S_{z}$, and $F, F^{\dagger}$ transform as a doublet under this $\mathrm{sl}_{2}$, and so form a Lie ideal. (We note that $(-1)^{i} f_{i}^{\dagger} f_{i}$ is the fermion number $=0,1$ at site $i$.) The Lie superalgebra is thus not semisimple, but is a semidirect product of these two, and can be viewed as the superalgebra of translations and $\mathrm{sl}_{2}$ rotations of the superplane with anticommuting coordinates $f, f^{\dagger}$. The associative (universal enveloping) algebra it generates, $\mathscr{A}_{1 \mid 1}$, is isomorphic to $U_{q}\left(\mathrm{sl}_{2}\right)$ (modulo the restriction to modules with integer $j$ ). Representations of this algebra acting in the spin chain can be easily constructed.

For the corresponding closed chain, generators like $F_{(2)}$ are lost, as the summation must be extended around the chain, and then anticommutation of $f_{i}$ and $f_{i^{\prime}}$ makes it vanish.

We emphasize that for open $\operatorname{gl}(n \mid n)$ chains with $n>1$, the symmetry algebra $\mathscr{A}_{n \mid n}$ is not an enveloping algebra of a finite-dimensional Lie superalgebra. For the closed chains, some symmetry operators of the open version are lost on closing the chain, for reasons similar to the case of $F_{(2)}$ above, but for general $n>1$ many operators remain. 


\section{Towards an associative-algebraic approach to (logarithmic) CFT}

It follows immediately from our analysis that the commutant algebra $\mathscr{A}_{m}$ is a symmetry of the low-lying spectrum of hamiltonians based on the Temperley Lieb algebra for any finite $L$. We now wish to take the limit $L$ going to infinity. It is not entirely clear how this can be done in a mathematically rigorous way, but roughly we want to take the eigenvectors of $H$ that have lowenergy eigenvalues, and we expect that the inner products among these vectors can be made to tend to some limits. Further, if we focus on long wavelength Fourier components of the set of $e_{i}$, then we expect their limits to exist, and their commutation relations to tend to those of the Virasoro generators $L_{n}\left(L_{n}+\bar{L}_{n}\right.$ in the closed chain case) [33], in the sense of weak convergence of matrix elements in this basis of low-energy eigenvectors. Then the modules over the TL algebra become modules over the UEA of the Virasoro, or possibly even a larger, algebra. (For the closed chains, two copies of the Virasoro algebra with generators $L_{n}, \bar{L}_{n}$ should eventually emerge.)

The symmetry (commutant) algebra in the continuum limit, which commutes with the Virasoro algebra, must be at least as large as that in the finite- $L$ chains. For the open chains, it appears that our commutant algebra [or $U_{q} s l_{2}$ in the spin-1/2 chain] does not become even larger in the limit. (In certain cases, such as the open or closed $m=2$ spin- $1 / 2$ chain, the symmetry algebra combines with the Virasoro algebra to form the $\mathrm{sl}_{2}$ level 1 current [affine Lie] algebra, but this is not the case in general [18].) In the continuum limit, the basis for the commutant algebra takes a similar form as on the lattice, and can now be written for the open cases using

$$
\begin{aligned}
& \widetilde{J}_{b_{1} b_{2} \cdots b_{k}}^{a_{1} a_{2} \cdots a_{k}}= \\
& \quad \int_{0<x_{1}<x_{2}<\cdots<1} \prod_{i=1}^{k} d x_{i} J_{b_{1}}^{a_{1}}\left(x_{1}\right) J_{b_{2}}^{a_{2}}\left(x_{2}\right) \cdots J_{b_{k}}^{a_{k}}\left(x_{k}\right)
\end{aligned}
$$

where the integration is over $0<x_{1}<x_{2}<\cdots<x_{k}<1$ (where $x L$ is position on the chain, and all operators are at the same time), $J_{b}^{a}(x)$ stands for the density of the generators $J_{i b}^{a}$ at $x$, and the contraction of any upper with a neighboring lower index is required to vanish as before. The integration domain in these expressions resembles that for the generators of the Yangian in an integrable system, but we emphasize again that our algebra is not the Yangian. The definition can be generalized using an arbitrary Jordan curve $C$ with ends on the boundary (even with both ends on the same boundary), with $J_{b}^{a}(x)$ replaced by the component of the divergenceless currents $J_{\mu b}^{a}$ $(\mu=1,2)$ normal to the curve, and the integrations are long the curve. These definitions also apply mutatis mutandis to the symmetry algebras of the closed chain, with an arbitrary closed Jordan curve $C$ in the most general form. These definitions for general curves ensure that the enlarged symmetry really commutes with conformal mappings of spacetime (which map curves $C$ onto one another), and generalize those for the global symmetry generators of $\mathrm{gl}(m+n \mid n)$, etc. [We note that while, unlike in theories with an affine Lie algebra, the Noether currents $J_{\mu b}^{a}$ do not possess a decomposition into purely holomorphic and anti-holomorphic parts [18], their flux across a curve is the Noether charge and is conserved and conformally invariant, and thus this is expected to hold for our operators also.]

We propose now that the use of the symmetry algebras (that commute with the full chiral algebra) can be a powerful organizing principle, even when semisimplicity of the algebraic structures is lost. These symmetries and structures can be usefully studied by starting with lattice models. 
The idea of gaining understanding of logarithmic CFT (LCFT) by studying lattice realizations in details has been around for a while, and put forward most recently (albeit without the algebraic connotations we consider crucial) in [34]. It does open a fruitful new route to progress. Indeed, the lattice equivalent of the reducible but indecomposable Virasoro representations in LCFT is the non semi-simplicity of the various algebras underlying the lattice models. [Examples include the Temperley-Lieb (TL) algebra or $U_{q}\left(\mathrm{sl}_{2}\right)$, neither of which is semi-simple when $q$ is a root of unity; such values are usually those of greatest interest.] A great deal of progress has been made in the mathematics literature in this area over the last fifteen years or so. Our strategy will be to exploit this progress and infer from it results about the LCFTs.

Indeed, it is a natural step to conjecture, from the lattice analysis, the corresponding structure in the continuum limit: the algebra of Hamiltonian densities goes over to the Virasoro algebra $[33,35]$, while the commutant goes over to an algebra of non-local charges. The general structure of the theory is one in which the Virasoro algebra commutes with a certain symmetry algebra, but the latter is not any kind of current algebra, and nor is it a Yangian. The structure of indecomposable representations in the lattice model goes over to the continuum limit, and we thus obtain information about the representations of the Virasoro algebra in our logarithmic theories. These representations are typically reducible but indecomposable with a diamond (or quartet) shape and made out of two "standard modules" connected by some "glue". They bear a lot of resemblance to the so-called staggered modules introduced abstractly in [36]. The full structure of the Hilbert space involves big indecomposables representations of Virasoro and of its commutant, and can be exhibited in the form of what we call a "staircase diagram" [6].

Although our approach does not so far yield much information on the detailed structure of the Virasoro indecomposable representations, it is enough to give access to the fusion rules for logarithmic theories. We work these out in detail in several cases in [6].

Acknowledgments: I thank the organizers of the Solvay Workshop for their kind invitation to this memorable event.

\section{References}

[1] F.D. Haldane et al. , Phys. Rev. Lett. 69 (1992) 2021.

[2] T. Deguchi, K. Fabricius and B. Mc Coy, J. Stat. Phys. 102 (2001) 701.

[3] P. Di Francesco, P. Mathieu, and D. Senechal, Conformal Field Theory (Springer, New York, 1997).

[4] V. Gurarie, Nucl. Phys. B 410, 535 (1993).

[5] N. Read and H. Saleur, "Enlarged symmetry algebras of spin chains, loop models and S matrices", cond-mat/0701259

[6] N. Read and H. Saleur, "Associative-algebraic approach to logarithmic conformal field theories", hep-th/0701117

[7] M.N. Barber and M.T. Batchelor, Phys. Rev. B 40, 4621 (1989).

[8] I. Affleck, J. Phys. Cond. Matt. 2, 405 (1990).

[9] H.N.V. Temperley and E.H. Lieb, Proc. Roy. Soc. (London) A 322, 251 (1971). 
[10] R. Baxter, Exactly Solved Models in Statistical Mechanics (Academic Press, New York, 1982).

[11] For our purposes, a module (modules are left modules unless stated otherwise) is the same as a representation in a vector space over $\mathbf{C}$.

[12] R.S. Pierce, Associative Algebras, Graduate Texts in Mathematics 88 (Springer-Verlag, New York, NY, 1982).

[13] F.W. Anderson and K.R. Fuller, Rings and Categories of Modules, Graduate Texts in Mathematics 13 (2nd Ed., Springer-Verlag, New York, NY, 1992).

[14] V.F.R. Jones, Invent. Math. 72, 1 (1983).

[15] F.M. Goodman, P. de la Harpe, and V.F.R. Jones, Coxeter Graphs and Towers of Algebras, MSRI 14 (Springer-Verlag, New York, NY, 1989), Ch. 2.

[16] P.P. Martin, Potts Models and Related Problems in Statistical Mechanics (World Scientific, Singapore, 1991).

[17] B.E. Sagan, The Symmetric Group: Representations, Combinatorial Algorithms, and Symmetric Functions, 2nd Ed., Graduate Texts in Mathematics 203 (Springer, New York, NY, 2001).

[18] N. Read and H. Saleur, Nucl. Phys. B 613, 409 (2001) [arXiv:hep-th/0106124].

[19] F.D.M. Haldane and D.J. Arovas, private communication (1991).

[20] These are reviewed in M. Freedman, C. Nayak, K. Shtengel, K. Walker, and Z. Wang, Ann. Phys. 310, 428 (2004) [arXiv:cond-mat/0307511].

[21] V. Chari and A. Pressley, A Guide to Quantum Groups (Cambridge University Press, Cambridge, 1994).

[22] C. Kassel, Quantum Groups, Graduate Texts in Mathematics 155 (Springer-Verlag, New York, NY, 1995).

[23] Strictly, here we mean the image of $U\left(\mathrm{sl}_{m}\right)$ in the finite-dimensional representations that are trivial under the center $\mathbf{Z}_{m}$ of $\mathrm{SU}(m)$.

[24] J.J. Graham and R.I. Lehrer, Invent. Math. 123, 1 (1996).

[25] A. Mathas, Iwahori-Hecke Algebras and Schur Algebras of the Symmetric Group, University Lecture Series Vol. 10 (American Mathematical Society, Providence, RI, 1999), and references therein.

[26] V.F.R. Jones, L'Enseignement Math. 40, 313 (1994).

[27] K. Erdmann and R.M. Green, arXiv:math.RT/9811017.

[28] P. Martin and H. Saleur, Commun. Math. Phys. 158, 155 (1993).

[29] P. Martin and H. Saleur, Lett. Math. Phys. 30, 189 (1994).

[30] G.H. Hardy and E.M. Wright, An Introduction to the Theory of Numbers, Fifth Edition (Clarendon, Oxford, 1979), Ch. XVI.

[31] I.A. Gruzberg, A.W.W. Ludwig, and N. Read, Phys. Rev. Lett. 82, 4524 (1999).

[32] The TL algebras for $m$ and $-m$ are isomorphic. Our definition of the algebra by relations (2.4) differs from some authors by a rescaling by $m$, which makes a difference only in the case $m=0$ (or $\infty$ ).

[33] W.M. Koo and H. Saleur, Nucl. Phys. B 426, 459 (1994).

[34] P. A. Pearce, J. Rasmussen and J.B. Zuber, "Logarithmic minimal models", hep-th/0607232. 
[35] G. Feverati and P. Pearce, Nucl. Phys. B 663, 409 (2003).

[36] F. Rohsiepe, "On Reducible but Indecomposable Representations of the Virasoro Algebra", hep-th/9611160. 